\title{
Power Fluctuation and Power Loss of Wind Turbine due to Wind Shear and Tower Shadow
}

\author{
Binrong WEN ${ }^{1} \quad$ Sha WEI ${ }^{1}$ Kexiang WEI ${ }^{2}$ Wenxian YANG ${ }^{3} \quad$ Zhike PENG $^{1}$ Fulei CHU ${ }^{4}$ \\ (1. Institute of Vibration, Shock and Noise, Shanghai Jiao Tong University, Shanghai China, \\ 200240;
}

2. Hunan Province Cooperative Innovation Center for Wind Power Equipment and Energy

Conversion, Xiangtan, China, 411100;

3. School of Marine Science and Technology, Newcastle University, Newcastle upon Tyne, UK

NE1 7RU;

4. Department of Mechanical Engineering Tsinghua University, Beijing, China, 100084)

\begin{abstract}
The magnitude and stability of the power output are two important indices for wind turbines. To estimate the capacity and quality of the power generated by a wind turbine, the relationships between power output and wind shear (WS) and tower shadow (TS) are discussed in this paper from the aspects of power fluctuation and power loss. WS and TS are described at first with a modification made to the TS model considering the cone-shaped tower of modern largescaled wind turbines. The power output of NREL 5MW reference wind turbine is calculated based on the modified Blade Element Momentum (BEM) Theory. Power fluctuation and power loss due to WS and TS are analyzed by theoretical calculation and case analysis, and the results indicate that TS is the main cause of power fluctuation while WS dominates in power loss. Power loss can be divided into wind farm loss and rotor loss, where wind farm loss is a constant while rotor loss is closely related to wind turbine control strategies. The results of this research can serve as a reference in accurate power estimation and strategies development to mitigate the fluctuation of aerodynamic loads and power output due to WS and TS.
\end{abstract}

Keywords Wind Turbine, Wind Shear, Tower Shadow, Power Fluctuation, Power loss

\section{Introduction}

Power generated by a wind turbine is more variable compared with conventional power generators due to both stochastic and periodic processes. Wind shear (WS) and tower shadow (TS) are two typical periodic effects. WS is used to describe the variation of wind speed with vertical elevation while TS demonstrates the reassigning of wind speed due to the presence of the tower. With the effects of WS and TS, the aerodynamic loads and power output of a wind turbine fluctuates periodically, and the fluctuation frequency was found to the 3 times of the rotation frequency of the rotor as for three bladed turbines ${ }^{[1,2]}$.

WS and TS, and their effects have been studied with different method from different aspects in previous publications. In 2002, Sorensen et $\mathrm{a}^{[3]}$ developed a wind model including WS and TS and other factors based on the power spectral description and the wind model was widely accepted in later studies ${ }^{[4-7]}$. Dai et $\mathrm{al}^{[8]}$ calculated the aerodynamic loads of large-scaled wind turbine with the modified Blade Element Momentum Theory (BEM) combined with dynamic stall model, and found that the aerodynamic loads were closely associated with TS, WS, dynamic stall and vibration of tower and blades.

Some researchers studied WS and TS through numerical simulation. Han et al ${ }^{[9]}$ carried out a numerical simulation analysis for a 3D steady fluid field around the wind turbine with WS considered and concluded that the effect of WS cannot be neglected for large-scaled wind turbines. Liu et a ${ }^{[10]}$ conducted a simulation about the 3D unsteady aerodynamic characteristics of a wind turbine with WS and TS considered by CFD method. The simulation pointed out that the value of WS exponent had a great impact of the aerodynamic loads of the wind turbine. When the WS exponent increased from 0.15 to 0.25 , the amplitude of the load fluctuation increased nearly $60 \%$. Investigation of Hughes et al ${ }^{[11]}$ showed that the period torque variation due to TS had a more significant influence on the power system stabilizer operation than the more distributed variation in wind velocity due to turbulence. Zhang et al ${ }^{[12]}$ investigated the influence of WS on vibration of wind turbine blade under fluid-structure interaction (FSI) via ANSYS simulation, and concluded that the contribution of WS to the displacement and the stress of the blade were larger than that of 
FSI.

Some researchers studied WS and TS from the aspect of the wake of a wind turbine. Wang et al ${ }^{[13]}$ studied the effects of WS and WS coefficients on the near wake of wind turbine and indicated that the torque of the blade as well as the characteristic of the blade near wake varied periodically due to WS. The axis, tangential, and radial velocities and the turbulence intensity were significantly affected by WS at the region of the blade near wake. Nilay et al ${ }^{[14]}$ investigated the impact of WS on the wake structure and the performance of wind turbine rotor with software AeroSIM+. The results showed that WS can create a complex wake structure with substantial asymmetries and then resulted in the fluctuation in the power and thrust of the blades.

In addition, since aerodynamic loads and power output fluctuate due to WS and TS, some strategies were proposed to mitigate the fluctuation, and the most popular one is individual blade pitch strategy, details can be found in references [15-19] ${ }^{[15-19]}$.

Most of the previous publications focused just on the fluctuation of aerodynamic loads and power output due to WS and TS. In fact, however, the effects of WS and TS on the power output are reflected in two aspects: power fluctuation and power loss. And the latter one is seldom discussed in the previous literatures. Liao et $\mathrm{al}^{[20]}$ mentioned the phenomenon of power loss but without any theoretical analysis. In this case, the relationships between power output and WS and TS were studied from the aspects of power fluctuation and power loss in this research. The results indicate that TS is the main cause of power fluctuation while WS dominates in power loss. Power loss can be divided into wind farm loss and rotor loss. Wind farm loss is a constant while rotor loss is closely related to wind turbine control strategies. NREL 5MW reference wind turbine was adopted to make the case analysis, and details about this turbine can be found in reference [21] ${ }^{[21]}$.

\section{Wind Shear and Tower Shadow}

\subsection{Wind Shear}

Wind shear (WS) model was developed to describe the variation in magnitude and direction of wind speed with the change of elevation. Wind speed usually increases with the vertical height since the influence of ground structures on the wind speed decreases. Power output and aerodynamic loads of the wind turbine oscillate periodically due to the different wind conditions encountered by each blade as it rotates during a complete cycle.

A widely accepted WS model is based on exponential distribution law, and is described as (1) ${ }^{[2]}$.

$$
V_{w s}(h)=V_{H}\left(\frac{h}{H}\right)^{\alpha}
$$

To simplify the analysis, formula (1) is converted into polar coordinate description

$$
V_{w s}(r, \theta)=V_{H}\left(1+\frac{r \cos \theta}{H}\right)^{\alpha}=V_{H}[1+w s(r, \theta)]
$$

Parameters in the formula are described in Fig 1 , where $V_{H}$ is the wind speed at the hub height $H ; r$ is the radial distance from the rotor axis, $\alpha$ is the empirical WS exponent, and $\alpha=0.1$ is assumed in this research; $h$ is the elevation of the analyzing point $(r, \theta)$, and $w s(r, \theta)$ is defined as wind shear coefficient, which indicates the disturbance in wind speed due to WS. Research results of Dolan ${ }^{[4]}$ showed that approximating $w s(r, \theta)$ by third truncated Taylor series expansion can reveal the essence of WS and simplify the calculating, as shown in (3).

$$
w s(r, \theta) \approx \alpha \frac{r}{H} \cos \theta+\frac{\alpha(\alpha-1)}{2}\left(\frac{r}{H}\right)^{2} \cos ^{2} \theta+\frac{\alpha(\alpha-1)(\alpha-2)}{6}\left(\frac{r}{H}\right)^{3} \cos ^{3} \theta
$$

Fig 2 describes the variation of wind shear coefficient $w s$ with azimuth $\theta$. It can be seen that wind speed encountered by a blade element (a given $r$ ) is nearly harmonic. And the variation of the ws increases with the increase of $r$ because blade elements farther from the rotor axis go through a larger elevation variation during a rotation cycle. The minimum $w s$ occurs at $\theta=180^{\circ}$, that is, when the blade is pointing downwards and the maximum ws occurs at $\theta=0^{\circ}$, that is, when the blade is pointing upwards. Take blade element at $r=56.17 \mathrm{~m}$ for example, the maximum and minimum $w s$ 
are $w s_{\max }=0.05174$ and $w s_{\min }=-0.08684$, respectively. The variation of $w s$ is $\Delta w s=w s_{\max }-w s_{\min }$ $=0.1386$, when the wind speed at hub height is $V_{H}=10 \mathrm{~m} / \mathrm{s}$, the wind speed variation is $\Delta V=V_{H} \cdot \Delta w s=$ $1.386 \mathrm{~m} / \mathrm{s}$, which is big enough to result in significant fatigue loads to shorten the lifespan of the blades.

Note that the average wind shear coefficient is not but smaller than zero, which is can be found from the absolute values of $w s_{\max }$ and $w s_{\min }$. Spatial average wind speed due to WS $\bar{V}_{w s}$ is defined as

$$
\bar{V}_{w s}=\frac{1}{\pi R^{2}} \int_{0}^{2 \pi} \int_{0}^{R} V_{H}[1+w s(r, \theta)] r d r d \theta=\left[1+\frac{\alpha(\alpha-1)}{8}\left(\frac{R}{H}\right)^{2}\right] V_{H}
$$

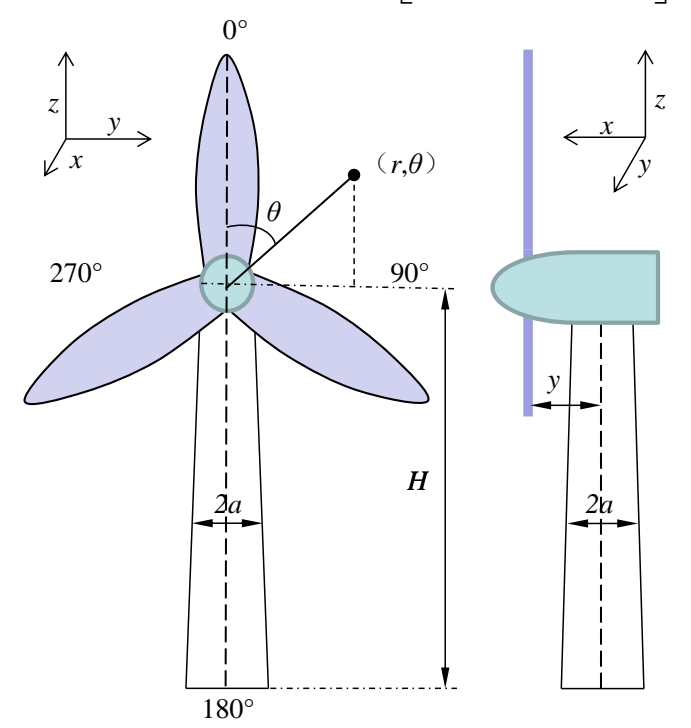

Fig 1 Schematic diagram of the wind turbine system

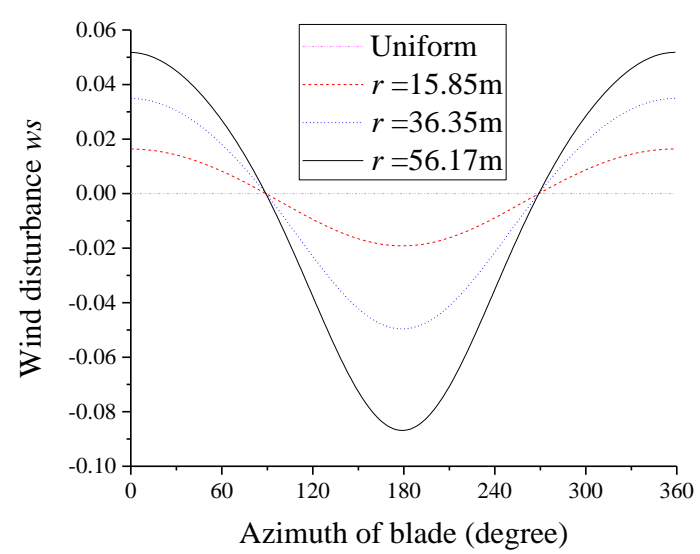

Fig 2 wind shear coefficient at different azimuth (uniform: uniform wind speed)

Because $\alpha$ is always less than 1 , so $\bar{V}_{w s}<V_{H}$, that is, spatial average wind speed due to WS is less than uniform wind speed. And the relative difference is defined as (5), which is a constant related to the WS exponent $\alpha$ and the structure parameter of the wind turbine $R / H$.

$$
\eta_{\bar{V}, w s}=\frac{\bar{V}_{w s}-V_{H}}{V_{H}}=\frac{\alpha(\alpha-1)}{8}\left(\frac{R}{H}\right)^{2}
$$

As for NREL 5MW reference wind turbine, $R=63 \mathrm{~m}, H=90 \mathrm{~m}$, which yield to $\eta_{\bar{V} \text {,ws }}=-0.0055$. With the effect of WS, the actual spatial average wind speed is slightly smaller than the uniform wind speed $V_{H}$, but the difference is small enough to allow uniform wind speed $V_{H}$ to serve as $\bar{V}_{w s}$ under circumstances where no strict calculation precision is required.

\subsection{Tower Shadow}

The distribution of wind speed is altered due to the presence of the tower. Wind directly in front of the tower decelerates while wind beside the tower accelerates. The phenomenon is named tower 
shadow effect (TS). It is widely believed that TS only affect the wind speed at region $90^{\circ}<\theta<270^{\circ}$ (see Fig 1$)^{[4,7]}$. The most prevalent TS model is based on potential flow theory, shown as $(6)^{[3,4]}$.

$$
V_{t s}(r, \theta)=V_{H}[1+t s(r, \theta)]
$$

Where $t s(r, \theta)$ is tower shadow coefficient, which is used to quantify the disturbance in wind speed due to TS, described as

$$
t s(r, \theta)=\left\{\begin{array}{lr}
a^{2} \frac{r^{2} \sin ^{2} \theta-x^{2}}{\left(r^{2} \sin ^{2} \theta+x^{2}\right)^{2}} \quad 90^{\circ}<\theta<270^{\circ} \\
0 \quad 0^{\circ}<\theta<90^{\circ}, 270^{\circ}<\theta<360^{\circ}
\end{array}\right.
$$

Where $x$ is the distance from blade origin to tower midline and $a$ is the tower radius (see Fig 1).

Formula (7) indicates that tower shadow coefficient $t s$ is proportional to the square of tower radius $a$. It is conceivable that a small change of $a$ will result in a great variation in $t s$. Considering that tower of modern large scale wind turbine tends to be cone-shaped, it is more reasonable to substitute the uniform $a$ in (7) with the exact $a$ value at different elevation. The exact tower radius at different location is shown as (8) and then formula (7) is modified into (9), where $a_{t}$ and $a_{b}$ are the tower radius at the top and the bottom, respectively.

Fig 3 shows the difference between formula (7) and (9), where $1.935 \mathrm{~m}$ and $3.000 \mathrm{~m}$ are the value of $a_{t}$ and $a_{b}$, respectively, and item Real $a$ means that different values of $a$ is used when the blade locates at different azimuth. The difference between these three curves verifies the necessity of modification of parameter $a$, especially for modern large-scaled wind turbines with a coned-shaped tower.

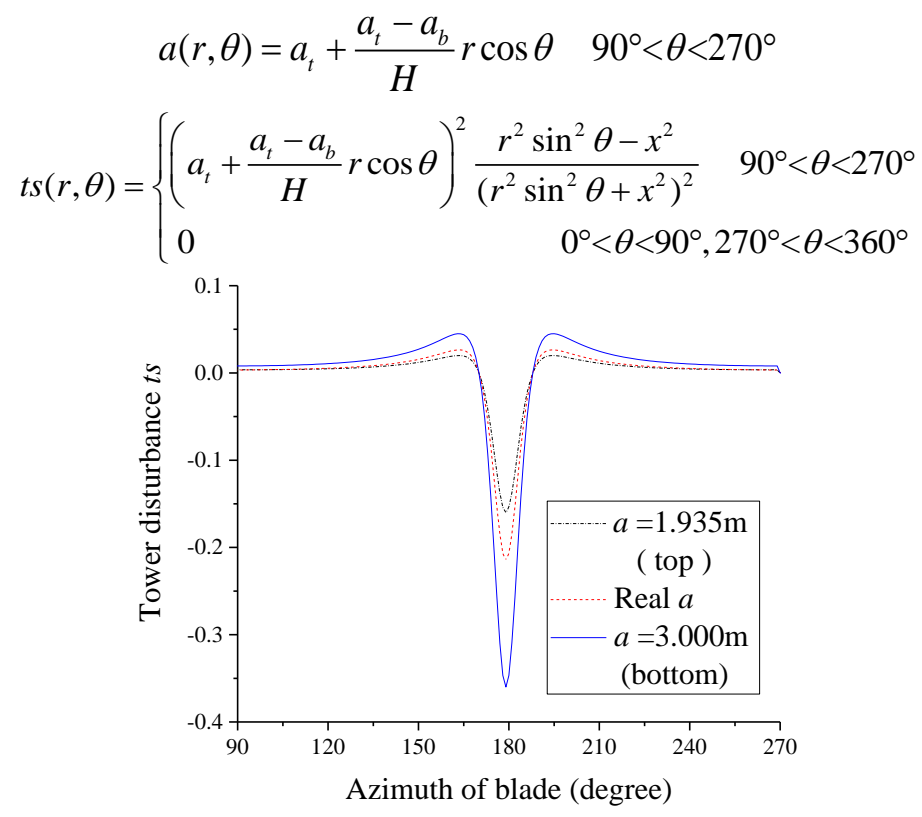

Fig 3 Variation of $t s$ with azimuth for different tower radius $a$

Fig 4 shows the variation of $t s$ with azimuth for different $r$, which indicates that TS reassigns the wind speed to shape an inverted cone. The whole space can be divided into three regions according to the different effects of TS on the wind speed. In case of $0^{\circ}<\theta<90^{\circ}$ or $270^{\circ}<\theta<360^{\circ}$, TS does not work with $t s=0$. When $180^{\circ}-\arcsin (x / r)<\theta<180^{\circ}+\arcsin (x / r)$, that is, directly in front of the tower, wind speed is significantly decreased. When $90^{\circ}<\theta<180^{\circ}-\arcsin (x / r)$ or $180^{\circ}+\arcsin (x / r)<\theta<270^{\circ}$, wind is accelerated due to TS with two peaks symmetric around $\theta=180^{\circ}$. As $r$ gets larger, the inverted cone narrows down with a smaller minimum to result in a more severe wind speed variation.

Take $r=61.63 \mathrm{~m}$ for example, the maximum and minimum $t s$ are $t s_{\max }=0.0353$ and $t s_{\min }=-0.2839$ respectively, so the $t s$ variation is $\Delta t s=t s_{\max }-t s_{\min }=0.3192$. When $V_{H}=10 \mathrm{~m} / \mathrm{s}$, the wind speed variation is $\Delta V=V_{H} \cdot \Delta t s=3.192 \mathrm{~m} / \mathrm{s}$. The wind speed fluctuation due to TS is almost three time of that due to WS (see section 1.1). This wind speed variation is serious enough to result in significant fatigue loads for turbine components and deteriorates the power output.

When $\theta=180^{\circ}$, $t$ r reaches the minimum, which is shown as formula (10). $t s_{\text {min }}$ depends on parameter 
$r$, and that is the consequence of the different tower radius at different elevation. If the tower radius is constant along the elevation, as formula (7), $t s_{\min }$ will be the same for different $r$, which is not the actual situation. It reveals the advantages of modified TS model (9) further.

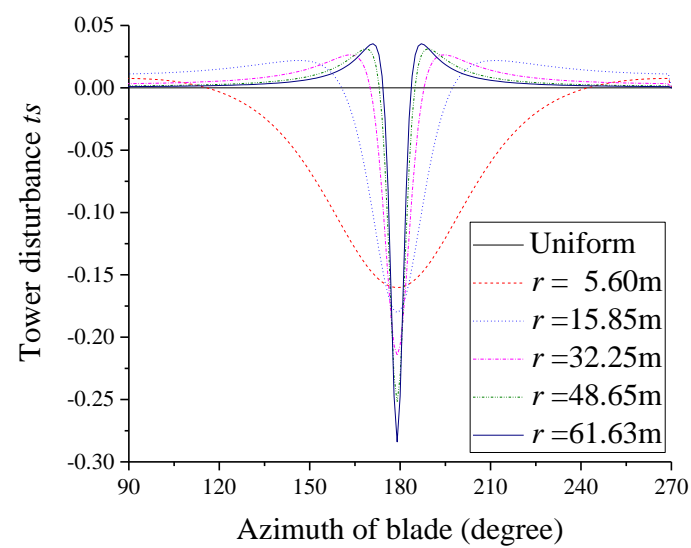

Fig 4 Variation of $t s$ with azimuth for different $r$

$$
t s_{\text {min }}(r)=\left.t s(r, \theta)\right|_{\theta=180^{\circ}}=\left(a_{t}+\frac{a_{b}-a_{t}}{H} r\right)^{2} \frac{1}{x^{2}}
$$

Similar to the analysis of WS, the spatial average wind speed due to TS is calculated as

$$
\bar{V}_{t s}=\frac{1}{\pi R^{2}} \int_{0}^{2 \pi} \int_{0}^{R} V_{t s}(r, \theta) r d r d \theta
$$

Substitute formula (6) and (9) into (11), we get

$$
\bar{V}_{t s}=V_{H}+\frac{V_{H}}{\pi R^{2}} \int_{\frac{\pi}{2}}^{\frac{3 \pi}{2}} \int_{0}^{R} t s(r, \theta) r d r d \theta
$$

The second term at the right hand side is the wind speed loss due to TS. To quantify the wind speed difference, the relative difference is defined as

$$
\eta_{\bar{v}, t s}=\frac{\bar{V}_{t s}-V_{H}}{V_{H}}=\frac{1}{\pi R^{2}} \int_{\frac{\pi}{2}}^{\frac{3 \pi}{2}} \int_{0}^{R} t s(r, \theta) r d r d \theta
$$

As for NREL 5MW reference wind turbine, $\eta_{\bar{v}, t s}=-0.0013$. Affected by TS, the actual spatial average wind speed is slightly smaller than the uniform wind speed $V_{H}$. Similar to WS, the difference is small enough to allow uniform wind speed $V_{H}$ to work as $\eta_{\bar{V}, t s}$ under circumstances where no strict calculation precision is required.

\section{Calculation model of power output}

To calculate the blade aerodynamic loads effectively, BEM theory, Generalized Dynamic Wake (GDW), and Computational Fluid Dynamics (CFD) have been developed. BEM is the oldest and most commonly used method with acceptable accuracy and the least computation attempts, which is especially suitable for steady aerodynamic analysis. Based on these considerations, BEM is adopted to calculate the power output of the wind turbine in this research.

A section of the rotor blade perpendicular to the pitch axis with length of $d r$ is taken for analysis. The velocities and forces acting on the airfoil are shown in Fig 5. Where $V_{0}$ is the absolute wind speed at the blade element; $\omega$ is the rotation speed of the rotor. Factors $a$ and $b$ are the axial induction factor and the tangential induction factor, respectively. $d R$ is the aerodynamic force acting on the airfoil, including lift force $d L$ and draft force $d D$. And $d N$ and $d Q$ are the axial and tangential force component of $d R$, respectively.

By applying blade element part of BEM, the lift force $d T$ and draft force $d Q$ can be written as

$$
d T=\frac{1}{2} \rho W^{2}\left(C_{l} \cos \varphi+C_{d} \sin \varphi\right) c d r
$$




$$
d Q=\frac{1}{2} \rho W^{2}\left(C_{l} \sin \varphi-C_{d} \cos \varphi\right) c d r
$$

Where $C_{l}$ and $C_{d}$ are the lift coefficient and drag coefficient of the airfoil, respectively. $c$ is the chord of the airfoil analyzed.

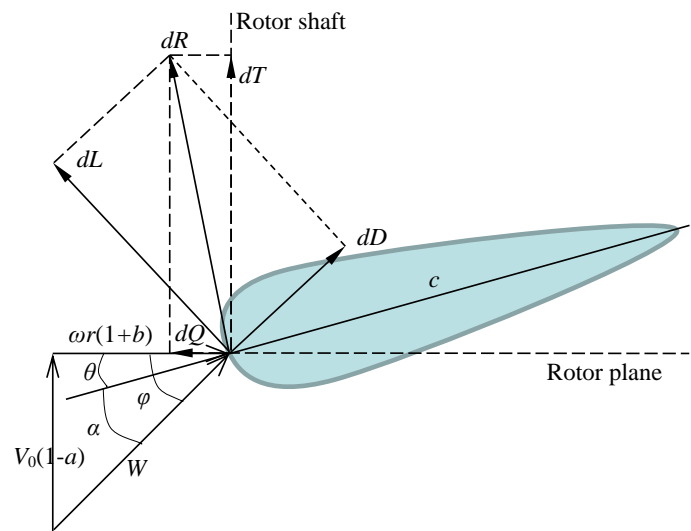

Fig 5 Velocities and forces on the airfoil

Incorporate the momentum part of BEM, which states that the thrust and torque extracted by each rotor annulus, shown as follows.

$$
\begin{gathered}
B d T=4 \pi r \rho V_{0}^{2}(1-a) a d r \\
B d Q=4 \pi r^{2} \rho V_{0} \omega(1-a) b d r
\end{gathered}
$$

Combine formula (14) (15) and (16) (17), the analytical solution of induction factors are

$$
\begin{gathered}
a=\left[1+\frac{4 \sin ^{2} \varphi}{\sigma\left(\mathrm{C}_{1} \cos \varphi+\mathrm{C}_{\mathrm{d}} \sin \varphi\right)}\right]^{-1} \\
b=\left[-1+\frac{4 \sin \varphi \cos \varphi}{\sigma\left(\mathrm{C}_{1} \sin \varphi-\mathrm{C}_{\mathrm{d}} \cos \varphi\right)}\right]^{-1}
\end{gathered}
$$

Where $\sigma$ is the local solidity, $\sigma=B c / 2 \pi r, B$ is the blade number of the turbine rotor.

The most important part of BEM is to get the accurate factor $a$ and $b$. Since there are so many initial assumptions of BEM theory, the calculation results is same how different with the experiment results, and then some correction models have been developed to improve the BEM, Prandtl model and Glauert correction included. Prandtl model serves to correct the induced velocity resulting from vortex shed near the blade tip and the hub of the rotor. And Glauert model was developed to correct the limitation of BEM when axial induction factor is greater than $0.4^{[22]}$.

Combining the initial BEM and Prandtl model and Glauert model, the induction factors and aerodynamic parameters can be got by the iteration algorithm, as shown in Fig 6.

With induction factors $a$ and $b$ calculated, and combined with formula (15), the power output of the blade element $d r$ is

$$
d P 1=\frac{1}{2} \rho W^{2}\left(C_{l} \sin \varphi-C_{d} \cos \varphi\right) c \omega r d r
$$

So the power output of a single blade, $P 1$, can be calculated by integrating $\mathrm{d} P 1$ along the blade span. And the total power output of the rotor is

$$
P=\sum_{i=1}^{B} P 1\left(\frac{2 \pi}{i}\right)
$$

When different wind speed model is considered, just replace $V_{0}$ with $V_{w s}, V_{t s}$ and $V$ during the calculating. $V$ means the actual wind speed affected by WS and TS at the same time.

The relationships between these wind speed models are demonstrated in Fig 7. Uniform wind model assumes that wind speed at different location is always the same. WS takes in to account the vertical change of wind speed while TS considers the horizontal change. With WS and TS considered at the same time, wind speed distribution is two-dimensional, which is the case of the actual wind speed $V$. 


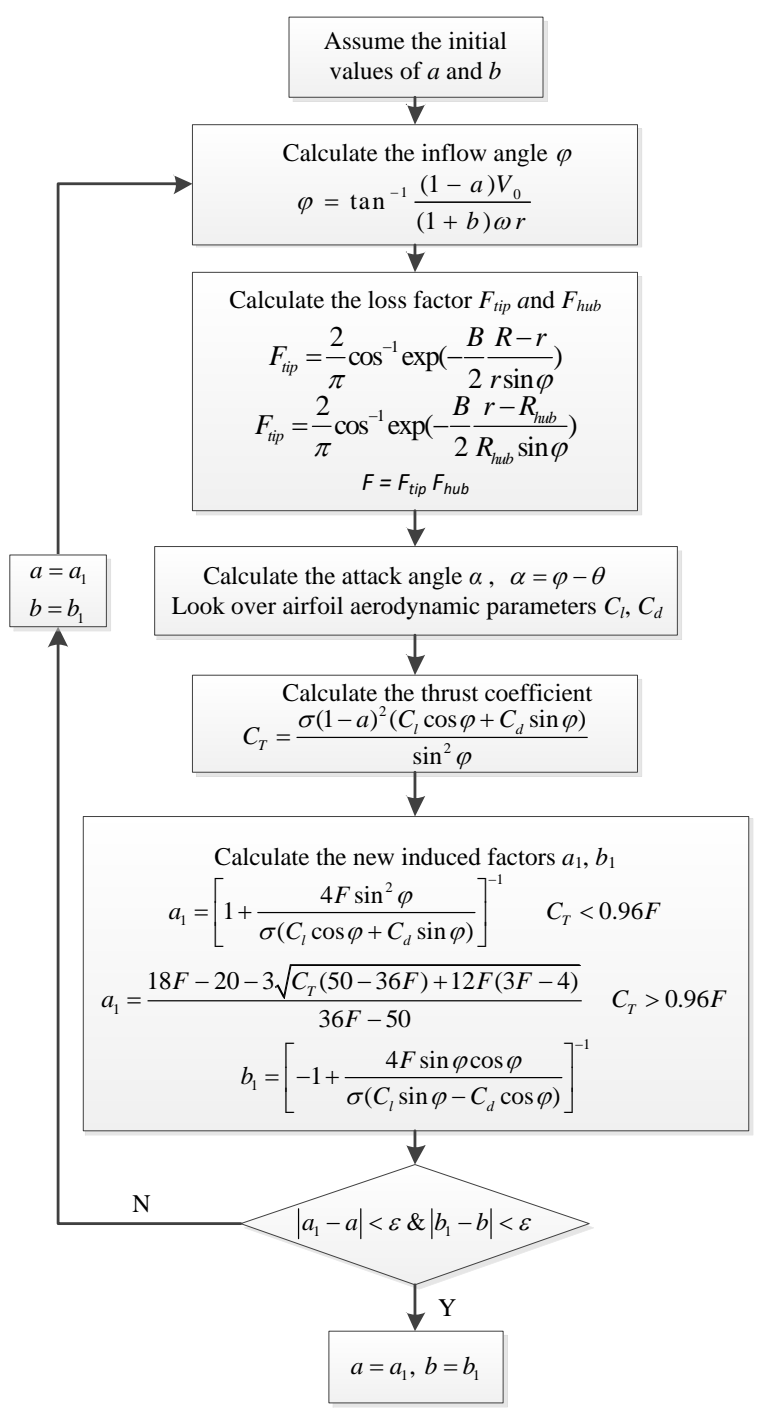

Fig 6 Calculation flow chart for induction factors

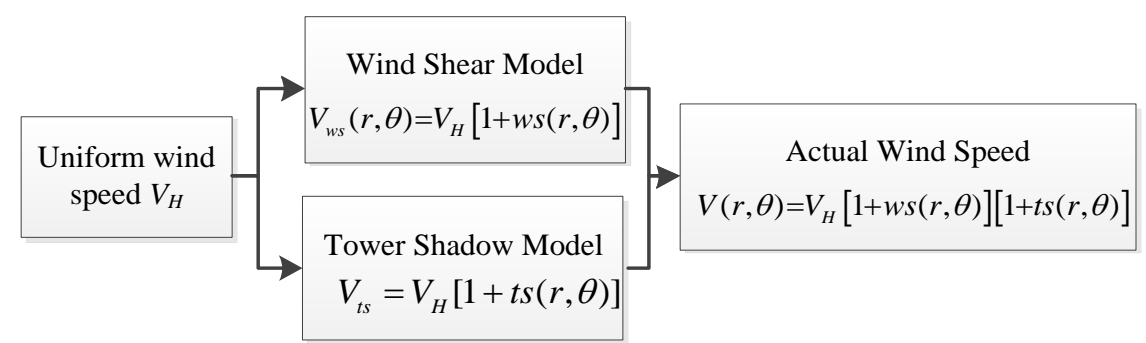

Fig 7 Relationships between different wind speed models

Fig 8 is the power output curve of NREL 5MW reference wind turbine calculated by different wind speed models based on the modified BEM theory. It can be seen that power loss occurs due to the effects of WS and TS compared with uniform wind speed model, especially at high wind speed. In engineering practice, however, the capacity of a wind turbine or a wind farm is estimated by uniform wind speed model. When estimating the capacity of a wind farm including lots of wind turbines, a remarkable overestimation occurs. In order to estimating the exact power output of the turbine and to reveal the essence of effects of WS and TS on power output, power fluctuation and power loss due to WS and TS will be discussed in detail in the next sections. 


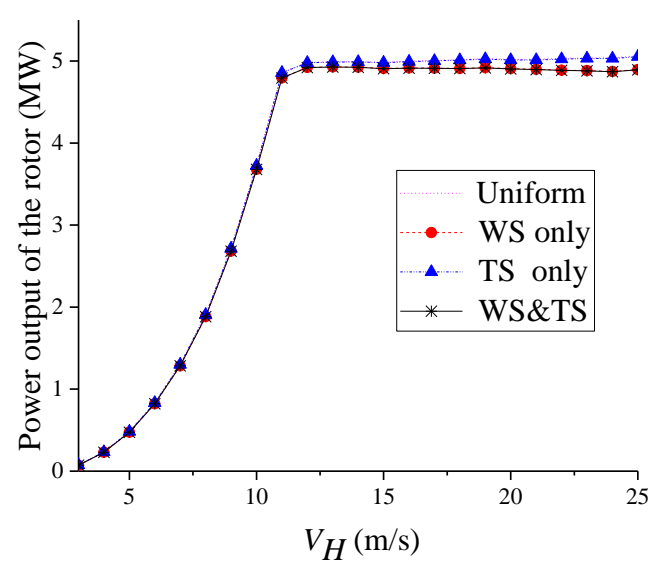

Fig 8 Power output of the rotor vs $V_{H}$

\section{Power fluctuation}

According to the calculation model of power output discussed in section 2, the power output of a single blade, $P 1$, of NREL $5 \mathrm{MW}$ reference wind turbine was calculated at uniform wind speed $V_{H}$ $=9 \mathrm{~m} / \mathrm{s}$ and Fig 9 shows the results. It can be seen that the power output fluctuation of a single blade is similar to that of wind speed (see Fig 2 and Fig 4).

Affected by WS only, $P 1$ varies sinusoidally with blade azimuth accompanied with a DC drop of $2.48 \%$. The peak-to-peak value is as large as $26.35 \%$. When TS works only, $P 1$ decelerates in a small region in front of the tower while accelerates besides the tower, and $P 1$ reaches the minimum value of 0.459 when the blade is pointing downwards. With WS and TS considered at the same time, the $P 1$ curve is always lower than the uniform one at the region of $90^{\circ}<\theta<270^{\circ}$ and coincide with that of WS when the blade is in the upper half plane. The minimum of $P 1$ occurs at $\theta=180^{\circ}$ with the value 0.3776 , which is much smaller than that of uniform wind speed and then leads to a great power drop to deteriorate the power capacity as well as the stability.

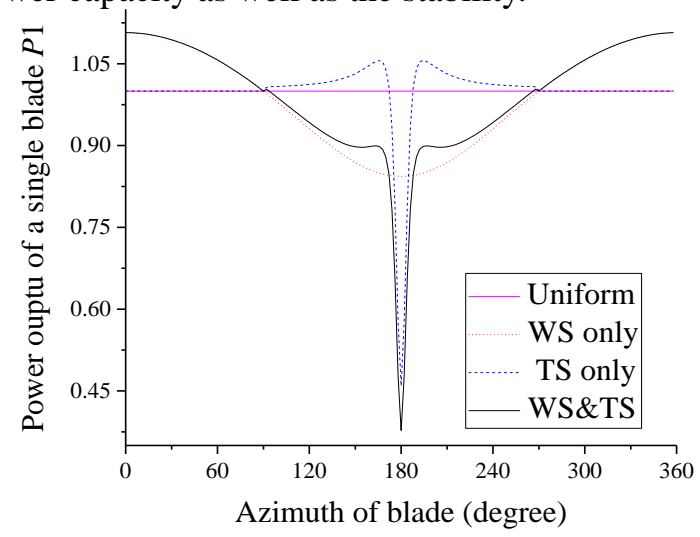

Fig 9 Power output of a single blade, $V_{H}=9 \mathrm{~m} / \mathrm{s}$

Rotor of modern large-scaled wind turbines tend to three bladed, so does NREL 5MW reference wind turbine. So according to (21), power output of the rotor is

$$
P=\sum_{i=1}^{3} P 1\left(\theta_{i}\right)=P 1(\theta)+P 1\left(\theta+120^{\circ}\right)+P 1\left(\theta+240^{\circ}\right)
$$

Fig 10 shows the power output variation of the rotor with azimuth under the same circumstance with Fig 9. It is obvious that $P$ fluctuates more frequently but with a smaller variation because the fluctuation of three blades compensate with each other.

With WS considered only, the power output of the rotor, $P_{w s}$, changes sinusoidally. Compared with the fluctuation of $P 1, P$ varies much less severely. The variation of $P_{w s}$ is $0.37 \%$, which is much smaller than that of $P 1(26.35 \%)$. But there is a still a DC drop of $1.23 \%$, which is about half of that of $P 1(2.48 \%)$. That is because three blades locate at different azimuth at the same time, so the effects of WS on different blades are different, which compensate with each other to mitigate the power 
fluctuation. It is imaginable that the stability of power output of the rotor can be improved further if the blade number is larger, but it is beyond the scope of this paper.

With TS considered only, the power output of the rotor, $P_{t s}$, fluctuates with the frequency three time of the rotation frequency. $P_{t s}$ is smaller than the uniform power only when a blade is approaching the lowest position, and for other cases $P_{t s}$ is always slightly larger than the uniform power. When $\theta=60^{\circ}, 180^{\circ}, 300^{\circ}$, that is, a blade of the rotor is pointing downward, $P_{t s}$ reaches the minimum with the value of 0.8197 . Compared with $P 1_{\text {min,ts }}$ ( value of 0.459 ), power loss of the whole rotor has been improved, and that can be accounted for by the compensation between three blades similar to WS. In addition, it can be seen that power fluctuation due to TS is much more severe than that due to WS. In another word, TS is the main cause of power fluctuation.

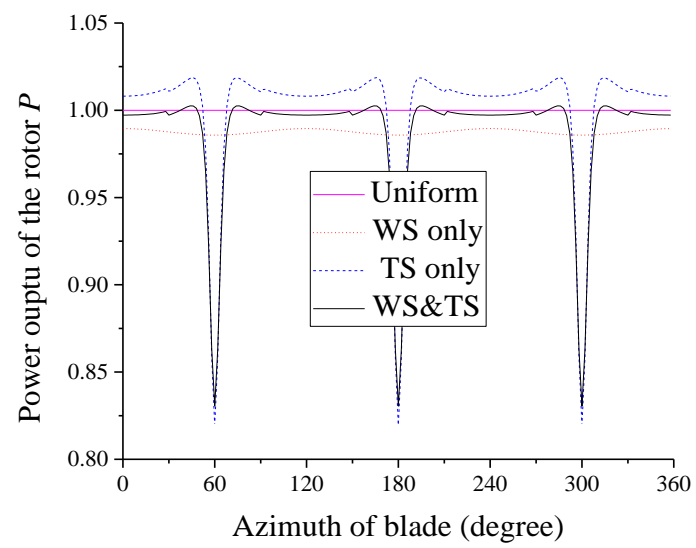

Fig 10 Variation of $P$ with azimuth, $V_{H}=9 \mathrm{~m} / \mathrm{s}$

With WS and TS considered at the same time, the power fluctuation has been mitigated further as a consequence of the compensation between WS and TS. Power output of the rotor, $P$, is nearly the same with uniform power except for cases of $\theta=60^{\circ}, 180^{\circ}, 300^{\circ}$. When $\theta$ approached value of $60^{\circ}$, $180^{\circ}$ and $300^{\circ}$, an inverted cone occurs, with the value of 0.8305 .

From the relative location of curves WS and WS \& TS in Fig 10, it can be seen that the average power output of the rotor is slightly smaller than that of the uniform one, and it will be discussed in detail in the following section.

\section{Power loss}

Power fluctuation due to WS and TS has been demonstrated in detail in section 3. In fact, however, WS and TS affect the power output of the rotor in the aspect of both power fluctuation and power loss. Using power output in the case of uniform wind speed as a reference, power loss is defined as

$$
\eta_{j}=\frac{P_{j}-P_{0}}{P_{0}}
$$

Where $j=w s, t s$, which means WS and TS, respectively. $P$ in this section is the average power output during a rotation period, and is simplified as power output. Subscript 0 means the case of uniform wind speed.

Power output of the rotor can be also calculated from

$$
\begin{gathered}
P_{j}=P_{w, j} \cdot C_{j} \\
P_{w, j}=\frac{1}{2} \rho \int_{0}^{2 \pi} \int_{0}^{R} V_{j}^{3} r d r d \theta
\end{gathered}
$$

Where $j=0, w s, t s ; P_{w, j}$ is the wind energy in the blade rotation span during unit time, which is defined as wind farm power; $C$ is the power capture coefficient and $\rho$ is the air density.

Formula (25) indicates that different wind speed model result in different wind farm power. Formulas (5) and (13) in section 2 show that the average wind speed of both WS model and TS model are less than uniform wind speed, and that means both WS and TS will result in wind farm power loss compared with power output in the case of uniform wind speed according to (25). This 
item of power loss is defined as wind farm loss.

On the other hand, power capture coefficient $C$ is thought to be tip speed ratio and pitch angle dependent in engineering practice, that is, $C=C(\lambda, \beta)$. In fact, however, from the principle of BEM, $C$ is the results of the superposition of all blade elements along the blade span. When different wind speed models are used, local wind speed of a single blade element varies to result in the change of $C$ value. The item of power loss due to the change of $C$ is defined as rotor loss with results of uniform wind speed model as reference.

\subsection{Wind farm loss}

Substitute formula (2) and (6) into (25), wind farm power under different wind speed model can be got, shown as follows.

$$
\begin{gathered}
P_{w, 0}=\frac{1}{2} \rho \pi R^{2} V_{H}^{3} \\
P_{w, w s}=\frac{1}{2} \rho \pi R^{2} V_{H}^{3}\left[1+\frac{3 \alpha(3 \alpha-1)}{8}\left(\frac{R}{H}\right)^{2}\right] \\
P_{w, t s}=\frac{1}{4} \rho \pi R^{2} V_{H}^{3}+\frac{1}{2} \rho V_{H}^{3} \int_{\frac{\pi}{2}}^{\frac{3 \pi}{2}} \int_{0}^{R}[1+t s(r, \theta)]^{3} r d r d \theta
\end{gathered}
$$

Consider that $t s$ is small, with only the first order approximation of $t s$ kept, (28) is simplified as

$$
P_{w, t s}=\frac{1}{2} \rho \pi R^{2} V_{H}^{3}+\frac{3}{2} \rho V_{H}^{3} \int_{\frac{\pi}{2}}^{\frac{3 \pi}{2}} \int_{0}^{R} t s(r, \theta) r d r d \theta
$$

According to the definition, wind farm loss due to WS and TS are

$$
\begin{gathered}
\eta_{w, w s}=\frac{P_{w, w s}-P_{w, 0}}{P_{w, 0}}=\frac{3 \alpha(3 \alpha-1)}{8}\left(\frac{R}{H}\right)^{2} \\
\eta_{w, t s}=\frac{\left(P_{w, t s}-P_{w, 0}\right)}{P_{w, 0}}=\frac{3}{\pi R^{2}} \int_{\frac{\pi}{2}}^{\frac{3 \pi}{2}} \int_{0}^{R} t s(r, \theta) r d r d \theta
\end{gathered}
$$

Formulas (30) and (31) indicate that wind farm loss due to both WS and TS, $\eta_{w, w s}$ and $\eta_{w, t s}$, are constant. Compared with the spatial average wind speed due to WS and TS, formula (5) and (13), it is found that

$$
\begin{aligned}
\eta_{w, w s} & =3 \eta_{\overline{\bar{V}}, w s} \\
\eta_{w, t s} & =3 \eta_{\overline{\bar{V}}, t s}
\end{aligned}
$$

Formulas (32) and (33) are coincident with the fact that wind farm power is third power of wind speed. Wind farm loss due to WS, $\eta_{w, w s}$, is related to the empirical wind shear exponent $\alpha$ and wind turbine structure parameter $R / H$, while wind farm loss due to TS, $\eta_{w, t s}$, depends on the parameters of the tower $a_{t}, a_{b}$, and $H$.

Case analysis shows that $\eta_{w, w s}=-1.2863 \%$ and $\eta_{w, t s}=-0.152 \%$, which is different to some degree from the three times relationship shown in (32) and (33) because the results are calculated by dispersion sum of all elements and inevitably with some error. Wind farm loss due to WS is much large than that of TS.

Actually, these losses can be explained perfectly from the wind speed model. The WS model, formula (3), shows that the WS works via item $\cos \theta, \cos ^{2} \theta$, and $\cos ^{3} \theta$. As for rotors consist of three blades, we get

$$
\begin{gathered}
\sum_{i=1}^{3} \cos \theta_{i}=\cos \theta+\cos \left(\theta+\frac{2}{3} \pi\right)+\cos \left(\theta+\frac{4}{3} \pi\right)=0 \\
\sum_{i=1}^{3} \cos ^{2} \theta_{i}=\sum_{i=1}^{3} \frac{\cos 2 \theta_{i}+1}{2}=\frac{3}{2} \\
\sum_{i=1}^{3} \cos ^{3} \theta_{i}=\sum_{i=1}^{3}\left(\frac{1}{4} \cos 3 \theta_{i}+\frac{3}{4} \cos \theta_{i}\right)=\frac{3}{4} \cos 3 \theta
\end{gathered}
$$


The $\cos \theta$ term yields a zero contribution while the $\cos ^{2} \theta$ term contributes a constant DC component which serves as the wind farm loss in the wind farm energy (see (30)) and DC drop in the power output analysis (see Fig 11). And the $\cos ^{3} \theta$ term is the root cause of sinusoidal change of power output due to WS as shown in Fig 10.

As for TS, formula (6), it is used to describe the reassigning of wind speed, or wind energy. In the ideal condition, it just reassigns the wind speed but without any energy loss. In fact, however, this model cannot satisfy the conservation of wind flux perfectly, which is a flaw of this TS model. Fortunately, this loss is small enough to ignore.

Since wind farm loss due to WS is much large than that of TS, the total wind farm loss, including loss due to WS and TS, can be equated with wind farm loss due to WS, and is simply named wind farm loss.

\subsection{Rotor loss}

As aforementioned, $C=C(\lambda, \beta)$ is assumed in engineering practice. And many models have developed to estimated power capture coefficient $C^{[24,25]}$. Jiang et al ${ }^{[26]}$ analyzed the limit power capture coefficient to serve as a reference in wind turbine design. In reality, power capture coefficient $C$ is closely connected with the wind speed model. The change of $C$ value results in rotor power loss. It is complicated to get a formula for the relationship between rotor power loss and wind speed model. Fortunately, however, the rotor loss can be got by total power loss minus wind farm loss as shown in the following case analysis.

Rotor power loss is defines as

$$
\eta_{C, j}=\frac{C_{j}-C_{0}}{C_{0}}=\frac{C_{j}}{C_{0}}-1
$$

Where $j=w s, t s$, and $C_{0}$ is the power capture coefficient for uniform wind speed model and serves as a reference.

Combine with formula (23) and (24), rotor loss can be calculated as

$$
\eta_{C, j}=\eta_{j}-\eta_{w, j}
$$

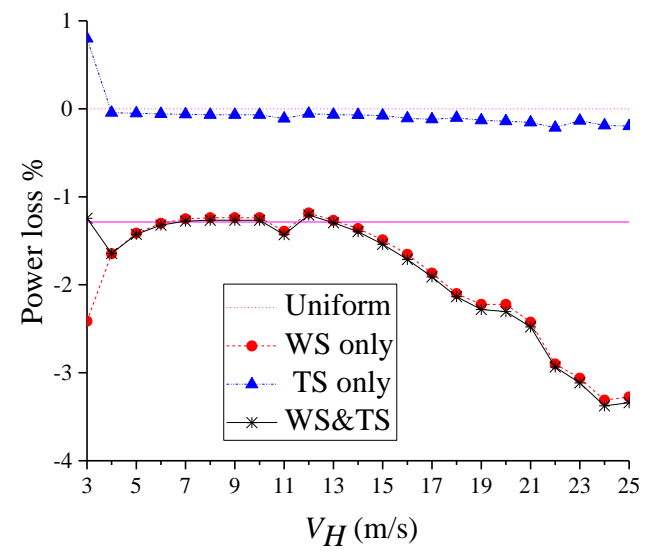

Fig 11 Variation of power loss with hub height wind speed

Power loss due to WS and TS at different hub height (uniform) wind speed is shown in Fig 11. It can be seen that power loss due to TS is small enough to be neglected compared with that due to WS. In other word, WS is the main cause of power loss. In that case, total power loss due to WS \& TS can be equated with total power loss due to WS, which is verified by the coincidence of curve WS only and WS \& TS. Note that power loss shown in the figure means the total loss, including the wind farm loss and the rotor loss. As aforementioned, wind farm loss is a constant, so the variation of total loss with wind speed is contributed by the rotor loss.

Fig 11 shows that power loss varies with wind speed, and it can be divided into three stages which is related to the control strategies of the wind turbine. As for NREL 5MW reference wind turbine, control strategies can be divided into three stages as well according to the wind speed. $3 \mathrm{~m} / \mathrm{s}<V_{H}<7.8 \mathrm{~m} / \mathrm{s}$ is the startup stage, where high torque capacity is provided; when 
$7.8 \mathrm{~m} / \mathrm{s}<V_{H}<11.4 \mathrm{~m} / \mathrm{s}$, variable speed control strategy works, which means the rotation speed of the rotor is controlled to maximize the power capture coefficient. When $V_{H}>11.4$, blade pitch controller is activated to change the blade pitch angle to keep the power output constant at the rated power of $5 \mathrm{MW}$.

The horizontal solid line with value of $-1.2863 \%$ in Fig 11 is the wind farm loss. During the startup stage $\left(3 \mathrm{~m} / \mathrm{s}<V_{H}<7.8 \mathrm{~m} / \mathrm{s}\right)$, power loss decreases with the wind speed getting stronger. Combined with formula (37), we know that in the startup stage power capture coefficient $C$ is approaching $C_{0}$ but always smaller than $C_{0}$ as $V_{H}$ increases. When $7.8 \mathrm{~m} / \mathrm{s}<V_{H}<11.4 \mathrm{~m} / \mathrm{s}$ where variable speed controller works, power loss is constant at the wind farm loss $(-1.2863 \%)$. It indicates that during the variable control stage, no rotor loss occurs, and the actual power capture coefficient $C$ equals $C_{0}$ and is the optimal value to maximize the power capture capacity. When $V_{H}>11.4 \mathrm{~m} / \mathrm{s}$, power loss increases with wind speed, and that is the consequence of blade pitch control which weakens the power capture capacity effectively. When wind speed $V_{H}$ reaches the cut-out speed $(25 \mathrm{~m} / \mathrm{s})$, rotor loss $\eta_{C}$ $=-2.0247 \%$. It is about 1.5 times of wind farm loss. When wind speed get larger, rotor loss takes up a larger and larger portion in the total power loss.

\section{Conclusions}

1) The tower radius $a$ is an important parameter for TS model, as for modern large-scaled wind turbine, it is necessary to utilize the real-time value of $a$ to improve the TS model.

2) Power output of rotor fluctuates with some power loss due to the effects of WS and TS.

3) TS effect is the main cause of power fluctuation. The fluctuation frequency is $B$ times of the rotation frequency, where $B$ is the blade number of the rotor. And the minimum power occurs when a blade is pointing downward.

4) WS effect dominates in power loss, which consists of wind farm loss and rotor loss. Wind farm loss is a constant related to wind shear exponent $\alpha$ and wind turbine structure parameter $R / H$. Rotor loss varies with wind speed and is closely related to the turbine control strategies.

\section{References}

[1] T Thiringer. Power quality measurements performed on a low-voltage grid equipped with two wind turbines [J]. IEEE Transactions on Energy Conversion, 1996, 11(3): 601-606.

[2] T Thiringer, J A Dahlberg. Periodic pulsations from a three-bladed wind turbine [J]. IEEE Transactions on Energy Conversion, 2001, 16(2): 128-133.

[3] P Sorensen, A D Hansen, P Andre, et al. Wind models for simulation of power fluctuations from wind farms [J]. Journal of Wind Engineering and Industrial Aerodynamics, 2002, 90:13811402.

[4] D S L Dolan, P W Lehn. Simulation model of wind turbine 3p torque oscillations due to wind shear and tower shadow [J]. IEEE Transactions on Energy Conversion, 2006, 21(3): 717-724.

[5] Y G Kong, J Gu, J Wang, Load analysis and power control of large wind turbine based on wind shear and tower shadow[J]. Journal of Southeast University, 2010, 40(1): 228-233.(in Chinese)

[6] Y G Kong, J Wang, J Gu,et al, Dynamics modeling of wind speed based on wind shear and tower shadow for wind turbine[J]. Acta Energiae Solaris Sinica, 2011, 32(8): 1237-1244. (in Chinese)

[7] S Das, N Karnik, S. Santoso Time-domain modeling of tower shadow and wind shear in wind turbines [J]. ISRN Renewable Energy, 2011, 2011:1-11.

[8] Dai J C, Hu Y P, Liu D S, et al. Aerodynamic loads calculation and analysis for large scale wind turbine based on combining BEM modified theory with dynamic stall model [J]. Renewable Energy, 2011, 36(3): 1095-1104.

[9] Z H Han, Y Li, J Ji. Numerical study on 3D steady flow of a $1.3 \mathrm{MW}$ wind turbine considering wind shear factor [J]. Journal of Chinese Society of Power Engineering, 2011, 31(10): 779-783. (in Chinese)

[10] L Liu, K C Shi, K Yang, et al. Effects of wind shear on the aerodynaimc load of wind turbine [J]. Journal of Engineering Thermophysics, 2010,31(10):1667-1670. (in Chinese)

[11] F M Hughes, O Anaya-Lara, G Ramtharan et al. Influence of tower shadow and wind turbulence on the performance of power system stabilizers for DFIG-basedwind farms [J]. IEEE 
Transactions on Energy Conversion, 2008, 23(2):519-528.

[12] J P Zhang, L Guo, H L Wu, et al. The influence of wind shear on vibration of geometrically nonlinear wind turbine blade under fluid-structure interaction [J]. Ocean Engineering, 2014, 84:1419.

[13] H P Wang, B Zhang, Q G Qiu. Numerical study of the effects of wind shear coefficients on the flow characteristics of the near wake of a wind turbine blade [J]. Proceedings of the Institution of Mechanical Engineers Part a-Journal of Power and Energy, 2016, 230(1): 86-98.

[14] N Sezer-Uzol, O Uzol. Effect of steady and transient wind shear on the wake structure and performance of a horizontal axis wind turbine rotor [J]. Wind Energy, 2013, 16(1): 1-17.

[15] Z X Xing, L Chen, W Li, et al, Pitch control method study on reducing the effects of tower shadow and wind shear[J]. Acta Energiae Solaris Sinica, 2013, 34(6): 916-923. (in Chinese)

[16] B Zhou, H J Gong, Z Y Zhen. The analysis of the pitch control of wind turbine by the influences of wind shear and tower shadow[J], Renewable Energy Resources, 2012, 30(1): 27-32. (in Chinese) [17] E A Bossanyi, G Hassan, S Lane. Individual blade pitch control for load reduction [J]. Wind Energy, 2003, 6(2): 119-128.

[18] H Namik, K Stol. Individual blade pitch control of floating offshore wind turbines [J]. Wind Energy, 2010, 13(1): 74-85.

[19] H Namik, K Stol. Individual blade pitch control of a floating offshore wind rurbine on a tension leg platform [C]//Proceedings of the 48th AIAA Aerospace Sciences Meeting Including the New Horizons Forum and Aerospace Exposition. 2010.

[20] M F Liao, K Xu, B Wu, et al. Effect of wind shear on wind turbine power[J]. Journal of Shenyang University of Technology, 2008, 30(2): 163-167. (in Chinese)

[21] J B S Jonkman, W Musial, G Scott. Definition of a 5-MW Reference Wind Turbine for Offshore System Development [J]. NREL/TP-500-38060, NREL: Golden, CO, 2009.

[22] P J Moriarty, A C Hansen. AeroDyn Theory Manual [M]. Salt Lake City, Utah, USA: National Renewable Energy Laboratory. 2005.

[23] Y Z Wu, H H Wang. Preliminary analysis on effect of wind shear on output power for large diameter wind turbine [J]. 2011, 6: 33-35. (in Chinese)

[24] Y Q Jia, A wind turbine simulator for wind generation research[J]. Acta Energiae Solaris Sinica,2004, 25(6): 735-739. (in Chinese)

[25] W W Li, D G Xu, W Zhang, et al. Research on wind turbine emulation based on DC motor [J]. IEEE Conference on Industrial Electronics and Applications, 2007, 5:2589-2593.

[26] H P Jiang. Power limit of horizontal axis wind turbine [J]. Journal of Mechanical Engineering, 2011, 47(10): 113-118. (in Chinese) 